\title{
EFFECT OF META SUBSTITUTION OF METHYL GROUP ON 2-HYDROXYPYRIDINE: SPECTROSCOPIC INVESTIGATION
}

\author{
A.K. Srivastava ${ }^{a}$ and S. Saxena ${ }^{b}$ \\ ${ }^{a}$ Department of Physics, Indian Institute of Technology Bombay, Mumbai-400076, Maharashtra, India \\ ${ }^{\mathrm{b}}$ Applied Physics Department, Sardar Vallabhbhai National Institute of Technology, Surat-395007, Gujarat, India \\ Email: pushpankit@gmail.com
}

Received 16 July 2019; revised 25 October 2019; accepted 31 October 2019

\begin{abstract}
We have reported here the detailed investigation of the effect of methyl group substitution on the meta-position of the 2-hydroxypyridine molecule. Resonance enhanced multiphoton ionization (REMPI), FT-IR and Raman spectroscopic techniques have been used for the experimental study of the molecules. Ab initio calculations were used for theoretical investigations of the molecules. The origin band of the molecules 3-methyl-2-hydroxypyridine (3M2HP) and 5-methyl-2-hydroxypyridine (5M2HP) was observed at 33830 and $34105 \mathrm{~cm}^{-1}$ in their REMPI spectroscopy, and the bands assigned as a $\pi \pi^{\star}$ transition state. The vibronic coupling of $n \pi^{\star}$ and $\pi \pi^{\star}$ transition states took place in $3 \mathrm{M} 2 \mathrm{HP}$, thus some low intense bands near the origin band of the molecule were observed in the REMPI spectrum. However, there was no such kind of bands in 5M2HP. The $\pi^{\star}-\sigma^{\star}$ hyperconjugation is responsible for the conformational change of the methyl group in 3M2HP upon excitation $\left(\mathrm{S}_{0} \rightarrow \mathrm{S}_{1}\right)$.
\end{abstract}

Keywords: methyl torsion, REMPI, ab initio, hyperconjugation, HOMO, LUMO

PACS: 31.15.A-, 31.50.-x, 33.15.Hp, 32.80.Rm

\section{Introduction}

The nitrogen heterocyclic molecules are very important in different kind of biological processes as well as in industrial applications, thus it needs attention to understand the photophysical and photochemical properties of these molecules. However, the fluorescence yield for these molecules is very low due to the close-lying excited states. In these molecules, there is a possibility of a close proximity of having another excited state $\left(n \pi^{\star}\right)$, that arises due to the presence of lone pairs, with the $\pi \pi^{*}$ excited state. In many heterocyclic molecules, these closely placed states result in strong mixing of the vibronic (vibrational and electronic) levels and thus affect the radiative as well as nonradiative energy decay properties of the excited states. Bickel et al. [1]] reported that the difference between the position of these two transition states $\left(\pi \pi^{*}\right.$ and $n \pi^{*}$ ) can be changed by substitution of a chemical group on any position of the molecule. Thus, the photophysical properties of these kinds of molecular systems depend on the electronic states and can be changed by any suitable substitution. Hence this kind of a molecular system can be used as modelled molecules to study the vibronic coupling of these transition states as well as the nature of molecular systems. 
The methyl group can affect the photophysical and photochemical properties of molecules and can also act as a probe for investigations. The coupling of the methyl group torsional motion with low frequency vibrations leads to the concept of mixing of two closely spaced electronic states. Lim and team [2] studied the energy states $n \pi^{*}$ and $\pi \pi^{*}$ of nitrogenous heterocyclic molecules and they concluded for the molecules quinoline and isoquinoline that the lowest energy state is an $n \pi^{*}$ state [2, 3]. Fischer [4, 5] worked on the spectroscopic study of isoquinoline vapour and found that the $n \pi^{\star}$ singlet state is slightly below the lowest energy $\pi \pi^{*}$ singlet state. The fluorescence excitation and dispersed fluorescence excitation spectroscopy of supersonic jet cooled isoquinoline and its methanol complex have been carried out by Felker and Zewail [ [6] and the coupling of $\pi \pi^{\star}$ and $n \pi^{*}$ states was observed for the molecules and its complexes [3, 6, 67. The methylisoquinolines were studied previously using laser induced fluorescence (LIF) and dipersed fluorescence (DF) excitation spectroscopic techniques [8]. The vibronic mixing of $n \pi^{\star}$ and $\pi \pi^{*}$ excited states was observed in methylated isoquinoline molecules and it was also reported that the $n \pi^{\star}$ state possessed longer life time than the $\pi \pi^{*}$ state.

The objective of this study is to understand the mechanism of the methyl torsional potential of the molecule in the ground electronic state as well as in the excited electronic states. However, the effect of methylation in the ground electronic state has been previously studied [9]. The focus is on the study of close-lying electronic states and the interactions due to the methyl group substitution on different positions in these methylated 2-hydroxypyridines. However, the interest is to understand the effect of different electronic environment on the methyl torsional behaviour and thus on the barrier potential of the molecule.

\section{Experimental section}

The resonance enhanced multiphoton ionization (REMPI) experiment was performed using a tunable dye laser which was pumped by a second harmonic of a pulsed Nd:YAG laser (Litron Nano Series Lasers, UK). For the ionization of molecules, the output from the tunable dye laser was frequency doubled using another second harmonic crystal, while the scanning of the dye la- ser frequency was done by using a stepper motor which was controlled by the developed LabVIEW program. The angle tuning of the second harmonic crystal was done manually, and then this final output was made to cross through a supersonic chamber, where the interaction with the sample takes place. The laser pulse and molecular jet were synchronized in time using two pulsed nozzle drivers (IOTA ONE Pulsed Driver from Parker Instrumentation) and a self-made microsecond pulsed delay generator. The opening of the pulsed nozzle is optimized for the cold molecular beam and maximum ionization signal. A convex lens of $8.0 \mathrm{~cm}$ focal length was used to focus the frequency doubled laser pulse inside the chamber, where the interaction takes place. The ionization signal, which was reflected by a plate termed as a repulser plate $(\mathrm{R})$, was collected in the direction perpendicular to both laser and molecular beams with the help of a channel electron multiplier (DR. SJUTS KBL 25RS). The signal from the channel electron multiplier is averaged (typically 128 pulses) and digitized using an optically triggered digital oscilloscope (TDS 1012B), and stored in a computer. The molecules 3-mehtyl2-hydroxypyridine and 5-methyl-2-hydroxypyridine were purchased from Sigma Aldrich and AlfaAesar chemical companies, respectively, and used without further purification. IR and Raman spectroscopy were performed using a FTIR-Imaging System (3000 Hyperion Microscope with Vertex 80 FT-IR System, Bruker, Germany) with a spectral resolution of FT-IR $0.2 \mathrm{~cm}^{-1}$ and a HR800UV confocal micro-Raman spectrometer (Horiba Jobin Yvon, France), respectively.

\section{Theoretical background}

$A b$ initio calculations have been used for the theoretical investigation of the molecules in the ground $\left(\mathrm{S}_{0}\right)$ as well as in the excited $\left(\mathrm{S}_{1}\right)$ electronic states. The geometry optimization has been performed along with the calculating barrier potential. The torsional potential in one dimension is given by $V(\tau)=\frac{V_{3}}{2}(1-\cos 3 \tau)+\frac{V_{6}}{2}(1-\cos 6 \tau)$, where $\tau$ is the torsional angle. All the $a b$ initio calculations for the ground as well as the excited electronic states were performed in Gaussian'09 [10] and the visualization of the molecular geometry and 
the molecular orbitals was carried out in the Gabedit software [11]. The procedure used for the theoretical study of the molecules in their ground $\left(\mathrm{S}_{0}\right)$ and excited $\left(\mathrm{S}_{1}\right)$ electronic states has been discussed in detail in our previous article [9, 12].

\section{Results and discussion}

\subsection{REMPI spectroscopy}

\subsubsection{3-Methyl-2-hydroxypyridine}

The REMPI spectrum of supersonic jet-cooled 3-methyl-2-hydroxypyridine (3M2HP) is shown in Fig. 1. The spectrum was recorded after $30 \mathrm{~min}$ of the release of argon gas. Broad as well as sharp spectral bands were observed in a range of 33800 to $33500 \mathrm{~cm}^{-1}$. The transition at $33830 \mathrm{~cm}^{-1}$ is assigned to the origin of the spectrum as there is no more low frequency below this. There is no appreciable change in the intensity of the peak whenever pressure of the gas increases. The excitation spectrum for the parent molecule 2-hydroxypyridine arises due to the $\pi \pi^{*}$ excitation [13] and no sign of $n \pi^{*}$ transition states arises in the spectrum. In a similar manner we assign the origin band at $33830 \mathrm{~cm}^{-1}$ to $\pi \pi^{*}$ transition. The origin band is red-shifted by $2294 \mathrm{~cm}^{-1}$ relative to the parent molecule indicating a change in excitation energy whenever the methyl group substitu-

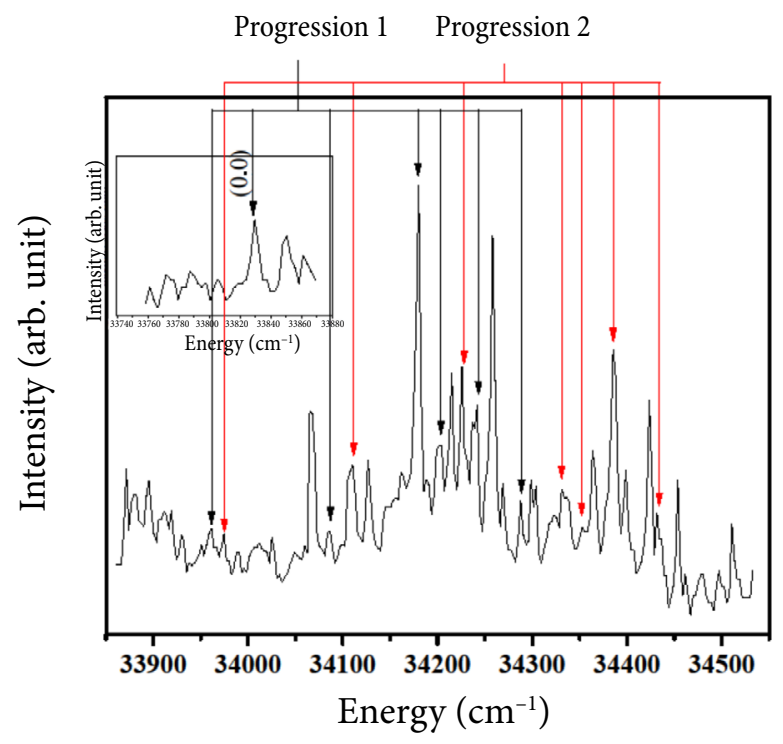

Fig. 1. REMPI spectrum of $3 \mathrm{M} 2 \mathrm{HP}$ after $30 \mathrm{~min}$ of release of argon gas. The spectrum is normalized with the laser intensity. tion takes place. There are several vibronic bands near the origin showing intense low frequency transitions. On the other hand, in the case of 2-hydroxypyridine no such weak band is observed in this region [13].

The possible reason for appearing of many low frequency bands near the origin could be the methyl internal rotational transitions, as it shows low frequency bands. But all the bands cannot be possible only because of the torsional transitions. Nimlos et al. recorded the excitation spectra for 2-hydroxypyridine and observed a number of transitions which cannot be assigned to any vibrational band which is associated with $\pi \pi^{\star}$ transitions [14]. Such transitions were also reported by Felker et al. [6] and explained with the mixing of $\pi \pi^{\star}$ and $n \pi^{\star}$ vibrational states which do not have a spacing characteristic of $\pi \pi^{*}$ transitions. The possible reason for appearing of many low frequency bands near the origin band could be the existence of a nearby $n \pi^{\star}$ state, and the vibronic coupling between the levels of $\pi \pi^{\star}$ and $n \pi^{\star}$. Table 1 shows the observed bands and their assignment.

Table 1. Assignment of the bands in the REMPI of 3M2HP.

\begin{tabular}{ccc}
\hline Energy, $\mathrm{cm}^{-1}$ & $\Delta v, \mathrm{~cm}^{-1}$ & Assignment \\
\hline 33830 & 0 & Origin $\left(\pi \pi^{\star}\right)$ \\
\hline 33961 & 131 & $2 \mathrm{e}$ \\
\hline 33974 & 144 & $v_{0}^{1}$ \\
\hline 34084 & 254 & $3 \mathrm{a} 1$ \\
\hline 34108 & 278 & $v_{0}^{1} 2 \mathrm{e}$ \\
\hline 34179 & 349 & $5 \mathrm{e}$ \\
\hline 34202 & 372 & $6 \mathrm{a} 2$ \\
\hline 34226 & 396 & $v_{0}^{1} 3 \mathrm{a} 1$ \\
\hline 34239 & 409 & $6 \mathrm{a} 1$ \\
\hline 34286 & 456 & $7 \mathrm{e}$ \\
\hline 34331 & 501 & $v_{0}^{1} 5 \mathrm{e}$ \\
\hline 34351 & 521 & $v_{0}^{1} 6 \mathrm{a} 2$ \\
\hline 34383 & 553 & $v_{0}^{\mathrm{v}} 6 \mathrm{a} 1$ \\
\hline 34421 & 591 & $v_{0}^{1} 7 \mathrm{e}$ \\
\hline
\end{tabular}

Close to the origin transition, a band at a separation of $21 \mathrm{~cm}^{-1}$ is observed. Assigning this band to the $2 \mathrm{e}$ torsional transition of the methyl group 
proclaimed the $3 \mathrm{a} 1$ and $4 \mathrm{e}$ bands around 53 and $83 \mathrm{~cm}^{-1}$, respectively. But there is no well-resolved band near the predicted value. Hence the second peak cannot be assigned to a torsional band. Also, assigning $21 \mathrm{~cm}^{-1}$ to a $2 \mathrm{e}$ band produces a barrier of a very low magnitude. But the barrier potential in the ground as well as in the excited states will be of the same order as there is no splitting of $0 \mathrm{a} 1,0 \mathrm{a} 1$ and 1e, 1e transitions for the origin band. Similar bands were reported for 1 MPY [15] in which it was assumed that this could be some anomalous band associated with the $n \pi^{*}$ transition.

The bands at 131 and $254 \mathrm{~cm}^{-1}$ from the origin band in the spectrum may be assigned to $2 \mathrm{e}$ and 3a1 torsional transitions, respectively. The potential parameters in the excited state can be obtained by fitting these bands. The excited state torsional parameters $\left(V_{3}^{\prime}=464 \mathrm{~cm}^{-1}, V_{6}^{\prime}=2 \mathrm{~cm}^{-1}\right.$, $F^{\prime}=5.3 \mathrm{~cm}^{-1}$ ) were obtained by the best fit of these observed bands. The calculated as well as experimentally observed torsional frequencies are presented in Table 2. The bands at 349, 372, 409 and $456 \mathrm{~cm}^{-1}$ can be assigned to $5 \mathrm{e}, 6 \mathrm{a} 2,6 \mathrm{a} 1$ and $7 \mathrm{e}$ torsional transition bands. There was no $4 \mathrm{e}$ band in the spectrum. The band at $278 \mathrm{~cm}^{-1}$ from the origin band could be due to the mixing of two vibrational modes. Hence, the band at $144 \mathrm{~cm}^{-1}$ was assigned to the second lowest vibrational mode $\left(v_{0}^{1}\right)$ in the excited state and can explain the presence of the band at $278 \mathrm{~cm}^{-1}$. There are many other vibrational modes in the spectrum due to the methyl torsional transitions which can be easily ex- plained by the combination of the other vibrational modes, as listed in Table 2 as progression 2. The bands at 396, 501, 521, 553 and $601 \mathrm{~cm}^{-1}$ are assigned to $3 \mathrm{a} 1,5 \mathrm{e}, 6 \mathrm{a} 2,6 \mathrm{a} 1$ and $7 \mathrm{e}$ torsion and vibration combination bands. The potential parameters used for the assignments of these combination bands are $V_{3}^{\prime}=472 \mathrm{~cm}^{-1}, V^{\prime}{ }_{6}=1 \mathrm{~cm}^{-1}$ and $F^{\prime}=5.3 \mathrm{~cm}^{-1}$.

\subsubsection{5-Methyl-2-hydroxypyridine}

The obtained REMPI spectrum of jet-cooled 5-methyl-2-hydroxypyridine (5M2HP) is shown in Fig. 2. The spectrum is recorded after $30 \mathrm{~min}$ of the release of the gas. One intense peak with few other peaks was observed in a spectral range of 33900 to $34550 \mathrm{~cm}^{-1}$. The observed spectrum was then normalized using the dye laser profile. The transition band at $34105 \mathrm{~cm}^{-1}$ has been defined as the origin band of the molecule. This is the $\pi \pi^{*}$ transition band. The characteristic of this band and the intensity pattern in the spectrum indicates an unchanged molecular conformation in the excited state compared to the ground state. The other bands are either vibrational bands or methyl torsional bands of the molecule. These bands are at 169, 267, $291,316,348$ and $384 \mathrm{~cm}^{-1}$ from the assigned origin $\left(34105 \mathrm{~cm}^{-1}\right)$. The assignment of the torsional band transitions 169, 267 and $291 \mathrm{~cm}^{-1}$ was identified as $3 \mathrm{a} 1,6 \mathrm{a} 2$ and $6 \mathrm{a} 1$ using the potential parameters $V_{3}=276 \mathrm{~cm}^{-1}, V_{6}=-50 \mathrm{~cm}^{-1}$ and $F=5.3 \mathrm{~cm}^{-1}$. Meanwhile, the band at $316 \mathrm{~cm}^{-1}$ matched with the band

Table 2. Assignment of the torsional transitions in the REMPI spectrum. Comparison between the experimentally observed and the calculated frequencies of a methyl group $\left(V_{3}^{\prime}=464 \mathrm{~cm}^{-1}\right.$, $\left.V_{6}^{\prime}=2 \mathrm{~cm}^{-1}, F^{\prime}=5.3 \mathrm{~cm}^{-1} ; V_{3}^{\prime}=472 \mathrm{~cm}^{-1}, V_{6}^{\prime}=1 \mathrm{~cm}^{-1}, F^{\prime}=5.3 \mathrm{~cm}^{-1}\right)$.

\begin{tabular}{|c|c|c|c|c|c|}
\hline \multicolumn{3}{|c|}{ Progression 1} & \multicolumn{3}{|c|}{ Progression 2} \\
\hline $\mathrm{S}_{0} \rightarrow \mathrm{S}_{1}$ & Cal., $\mathrm{cm}^{-1}$ & Obs., $\mathrm{cm}^{-1}$ & $\mathrm{~S}_{0} \rightarrow \mathrm{S}_{1}$ & Cal., $\mathrm{cm}^{-1}$ & Obs., $\mathrm{cm}^{-1}$ \\
\hline $1 e-2 e$ & 136.2 & 131 & $1 e-2 e$ & 137.2 & 134 \\
\hline $0 \mathrm{a} 1-3 \mathrm{a} 2$ & 136.4 & - & $0 \mathrm{a} 1-3 \mathrm{a} 2$ & 137.4 & - \\
\hline $0 \mathrm{a} 1-3 \mathrm{a} 1$ & 254 & 254 & $0 \mathrm{a} 1-3 \mathrm{a} 1$ & 257 & 252 \\
\hline $1 e-4 e$ & 257 & - & $1 e-4 e$ & 259 & - \\
\hline $1 e-5 e$ & 349 & 349 & $1 e-5 e$ & 353 & 357 \\
\hline $0 a 1-6 a 2$ & 369 & 372 & $0 \mathrm{a} 1-6 \mathrm{a} 2$ & 372 & 377 \\
\hline $0 a 1-6 a 1$ & 410 & 409 & $0 a 1-6 a 1$ & 414 & 409 \\
\hline $1 e-7 e$ & 451 & 456 & $1 e-7 e$ & 455 & 457 \\
\hline
\end{tabular}




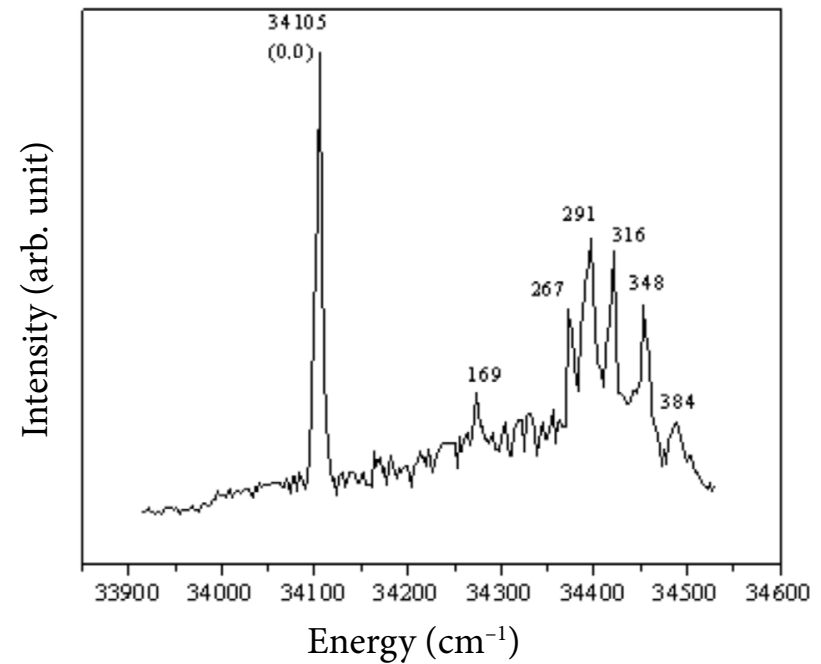

Fig. 2. REMPI spectrum of 5M2HP after $30 \mathrm{~min}$ of the release of helium gas. The spectrum is normalized with the laser intensity.

transitions for 3-methyl-2-hydroxypyridine. Hence, this band can be assigned to the vibration corresponding to the 3-methyl-2-hydroxypyridine molecule. The other bands at 348 and $384 \mathrm{~cm}^{-1}$ are assumed as some other vibration.

Table 3. Assignments of the bands in 5-methyl-2-hydroxypyridine $\left(V_{3}^{\prime}=276 \mathrm{~cm}^{-1}, V_{6}^{\prime}=-50 \mathrm{~cm}^{-1}\right.$ and $\left.F^{\prime}=5.3 \mathrm{~cm}^{-1}\right)$.

\begin{tabular}{ccc}
\hline Energy, $\mathrm{cm}^{-1}$ & $\Delta v, \mathrm{~cm}^{-1}$ & Assignment \\
\hline 34105 & 0 & Origin $\left(\pi \pi^{*}\right)$ \\
\hline 34274 & 169 & $3 \mathrm{a} 1$ \\
\hline 34372 & 267 & $6 \mathrm{a} 2$ \\
\hline 34396 & 291 & $6 \mathrm{a} 1$ \\
\hline 34421 & 316 & - \\
\hline 34453 & 348 & - \\
\hline 34489 & 384 & - \\
\hline
\end{tabular}

\subsection{IR and Raman spectrum}

\subsubsection{3-Methyl-2-hydroxypyridine}

For the assignments of the obtained vibrational as well as torsional bands in the ground electronic state, infrared (IR) and Raman spectroscopic techniques were analysed. The FT-IR and Raman spectrum of the molecule in the condensed phase is shown in Figs. 3 and 4, respectively. The spectral resolution in the far IR region is very high compared to that in the mid IR region, thus there is a minimum discrepancy in few of the band po-

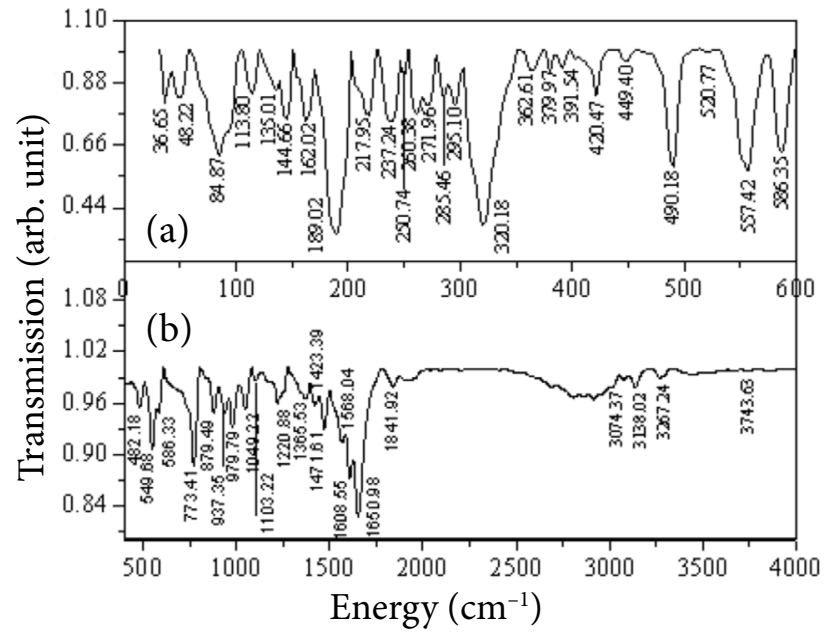

Fig. 3. IR spectra of $3 \mathrm{M} 2 \mathrm{HP}$ in (a) the far IR and (b) the mid IR region.

sitions and their relative intensity such as 490 and $557 \mathrm{~cm}^{-1}$ in Fig. 3(a). However, the main interest is to observe the low frequency bands near the origin due to the methyl torsion motion. The calculated frequencies with their relative IR intensity and Raman activity are listed in Table ST1 as supplementary material (Appendix 1) under the harmonic approximation with the optimized geometry in the B3LYP/TZVP level of theory. Few of the calculated low frequency bands are in close proximity with the experimentally observed bands in Raman and FT-IR. The band at $126 \mathrm{~cm}^{-1}$ is the pure torsional band of the molecule in the ground state which can also be seen in the Raman spectrum at $107 \mathrm{~cm}^{-1}$. While the bands

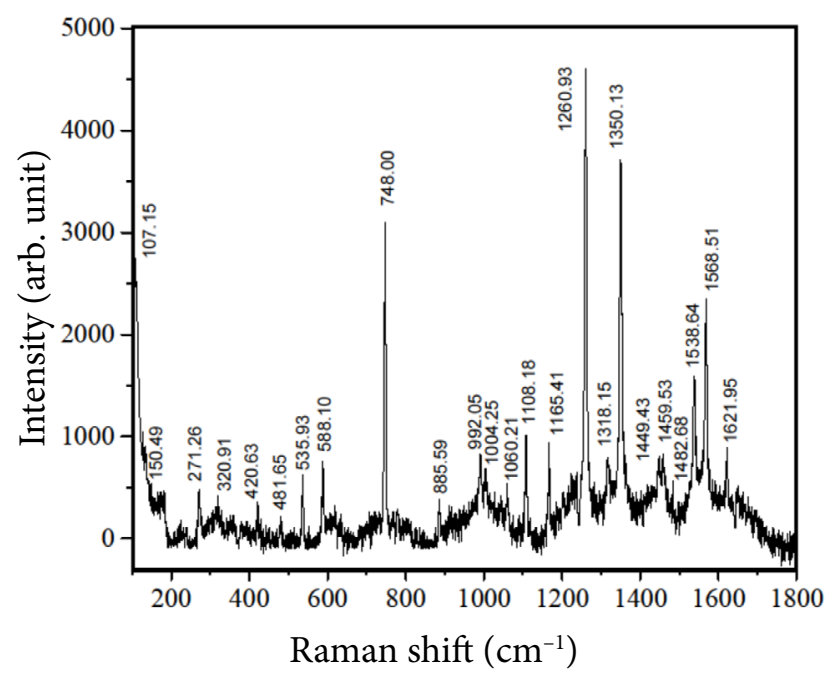

Fig. 4. Raman spectrum of 3-methyl-2-hydroxypyridine. 
at 150 and $271 \mathrm{~cm}^{-1}$ in the Raman spectrum can also be seen in the FT-IR spectrum at 162 and $272 \mathrm{~cm}^{-1}$, respectively. These low frequency bands are obtained due to the torsional motion of the ring frame and close to the theoretically obtained bands at 163 and $273 \mathrm{~cm}^{-1}$, respectively. The molecule has 15 atoms hence 39 fundamental frequencies are present. Among these 39 fundamental frequencies, 11 are in-plane and 28 are out-of-plane vibrational modes. For all the bands strong mixing of various vibrational modes was observed. However, the first three low frequency vibrations show torsional characteristics.

Figure 5 shows six low frequency vibrations in the ground electronic state of the molecule in which arrows represent the atomic displacements. The lowest frequency was at $126 \mathrm{~cm}^{-1}$. This is because of the torsional motion of the methyl group. The next two calculated low frequencies are at 163 and $273 \mathrm{~cm}^{-1} \cdot 163 \mathrm{~cm}^{-1}$ is observed as a mixture of various torsional motions of the ring frame with the $\mathrm{C}_{3} \mathrm{C}_{8}$ torsion, while $273 \mathrm{~cm}^{-1}$ is obtained because of the mixing of the torsional motion and the bending/wagging motion of the ring frame. These two frequencies are identified in the observed bands in IR and in Raman spectra. However, there was no band at $349 \mathrm{~cm}^{-1}$ in the Raman or the IR spectrum as it was present in the REMPI spectra of the molecule. There was no analogous frequency in the calculation. On the basis of these observations, this band was assigned to the torsional 'e' progression (5e).

\subsubsection{5-Methyl-2-hydroxypyridine}

The FT-IR and Raman spectrum of 5-methyl-2-hydroxypyridine is shown in Figs. 6 and 7 , respectively. The discrepancy, as discussed previously, is also observed in Fig. 6(a) for 500 and $527 \mathrm{~cm}^{-1}$ due to the spectral resolution. The $\mathrm{B} 3 \mathrm{LYP} /$ TZVP level of theory has been used to calculate the fundamental frequencies, their relative IR intensity and Raman activity under the harmonic approximation with the optimized geometry, and it is listed in Table ST2 as supplementary material (Appendix 2).

39 fundamental frequencies were obtained for the molecule in which mixing of various vibrational bands was observed as in 3M2HP. There

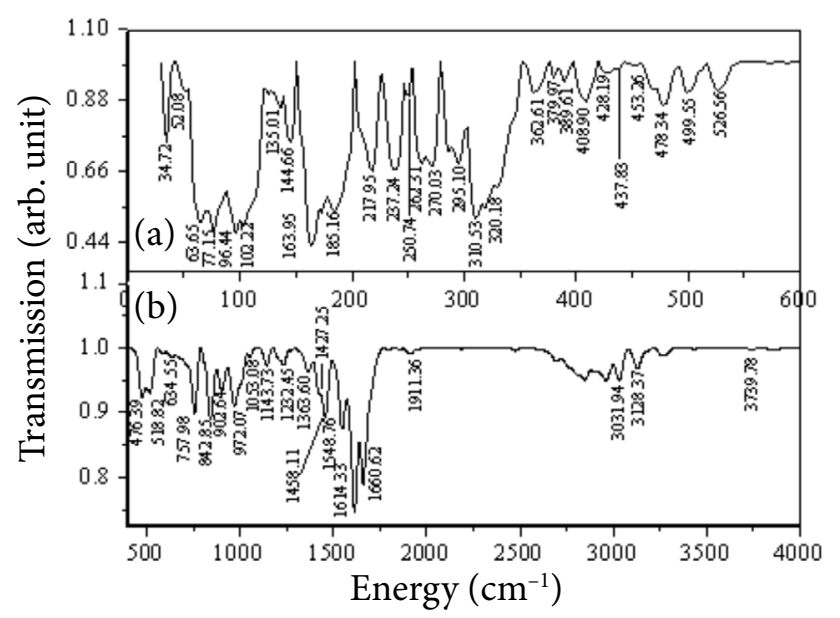

Fig. 6. IR spectra of 5M2HP in (a) the far IR and (b) the mid IR region.
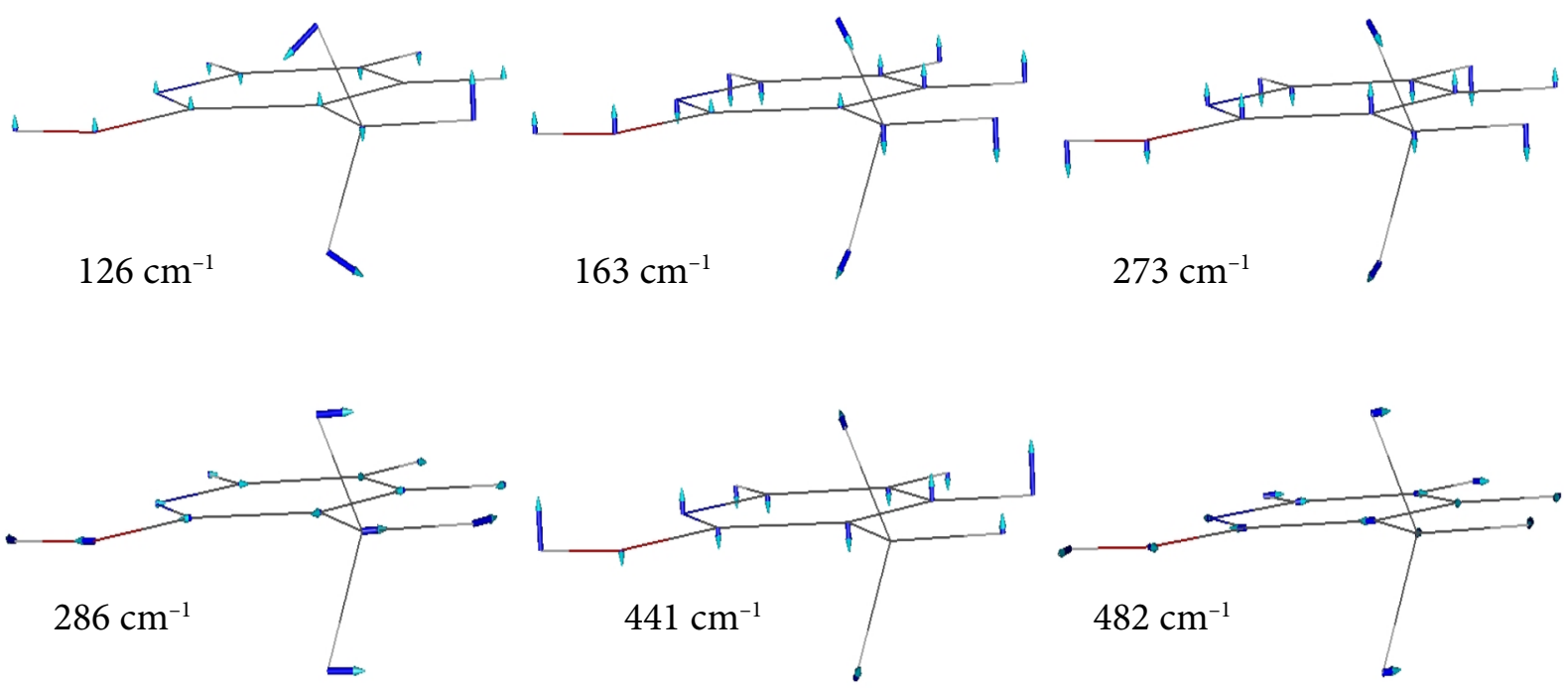

Fig. 5. First six low frequency vibrations in the ground electronic state of 3M2HP. 


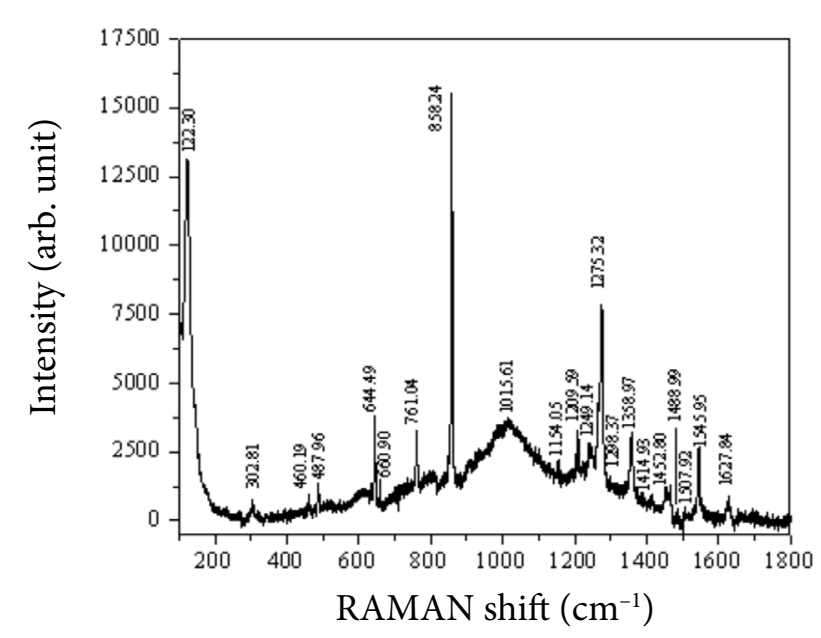

Fig. 7. Raman spectrum of 5-methyl-2-hydroxypyridine.

were no such low frequency vibrational bands as in 3-methyl-2-hydroxypyridine. The first two calculated frequencies were obtained as mixing of the vibrational motions. A low frequency at $74 \mathrm{~cm}^{-1}$ was observed as mixing of the torsional characteristic of the methyl group and the out-ofplane wagging/bending motion of the ring frame, while the band at $141 \mathrm{~cm}^{-1}$ was due to the mixing of the torsional motion of the ring as well as its out-of-plane wagging/bending motion.

\subsection{Theoretical study: Ab initio calculations}

\subsubsection{3-Methyl-2-hydroxypyridine}

The intensity pattern observed from the REMPI spectrum suggests the change in the methyl group position upon excitation from the ground electronic state to the excited electronic state $\left(\pi \pi^{\star}\right)$. The torsional potential due to the methyl group internal motion was estimated with the observed $5 \mathrm{e}$ band. Further investigation for the conformational study and the torsional potential in the excited state was carried out using the B3LYP/TZVP level of theory. The optimized geometry in the excited state is shown in Fig. 8(a). In the obtained minimum energy conformation of the molecule, the ring frame is always planar and the internal rotation of the methyl group was observed. The torsional angle dependence of the potential energy curve for the excited state $\left(\mathrm{S}_{1}\right)$ is shown in Fig. 8(b).

The curve was extracted from the ground state potential energy and the calculated excitation (a)

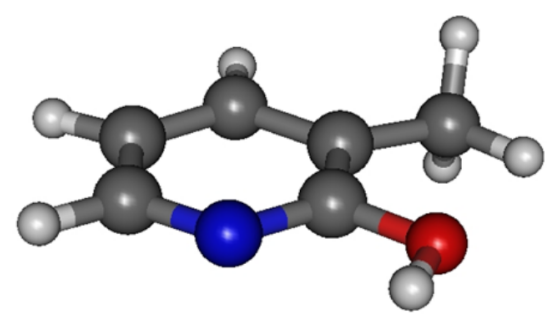

(b)

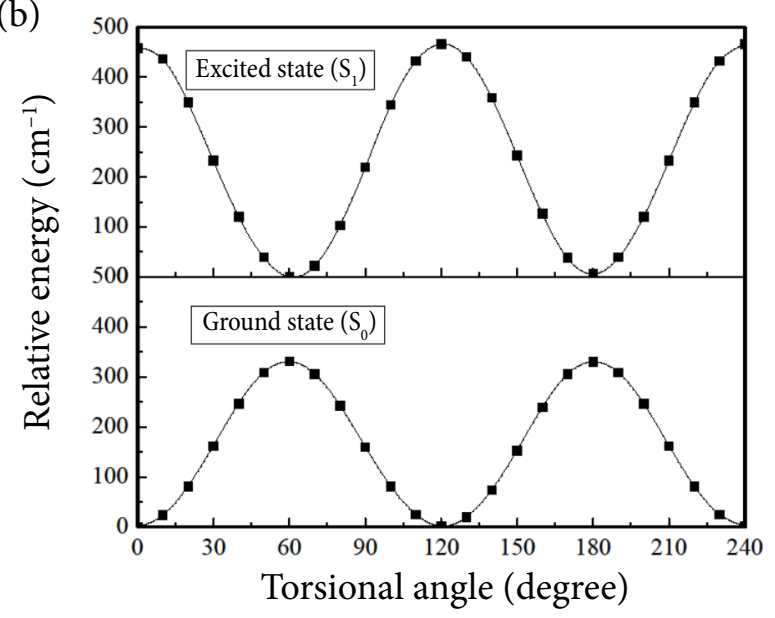

Fig. 8. (a) The optimized geometry and (b) the torsional angle dependence of the potential barrier curve for 3-methyl-2-hydroxypyridine using the B3LYP/ TZVP level of theory.

energies for every torsional angle. The calculated value of the three-fold potential term $V_{3}$ was found to be $464 \mathrm{~cm}^{-1}$ and matched with the experimentally observed value. It can also be seen from the potential curve that the potential minimum has been shifted by $60^{\circ}$ in the excited state relative to the ground state. This is in agreement with the result obtained from the optimized geometry and from the observed REMPI spectrum. The change in the methyl group conformation and the magnitude of the torsional potential barrier in 3-methyl-2-hydroxypyridine upon excitation $\left(\mathrm{S}_{0} \rightarrow \mathrm{S}_{1}\right)$ were explained using the $\pi^{\star}-\sigma^{\star}$ hyperconjugation [15]. The $\pi^{\star}-\sigma^{\star}$ hyperconjugation in the LUMO at the top of the barrier conformation stabilizes these in the ground as well as in the excited states. The orbital contour diagram for 3-methyl-2-hydroxypyridine in the HOMO and LUMO in the minimum and top of the barrier conformation is shown in Fig. 9. The $\pi^{\star}-\sigma^{\star}$ hyperconjugation was observed only in the 0 degree conformation in the LUMO. This signifies the change in conformation of the methyl group 


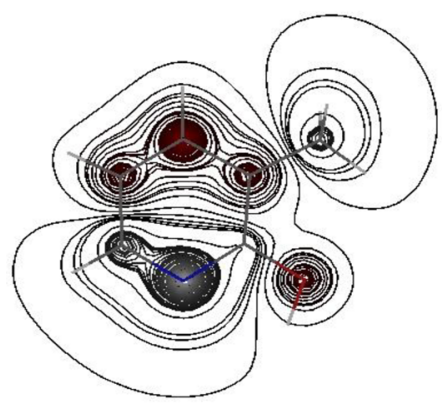

HOMO

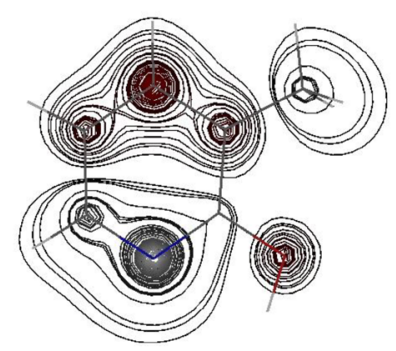

0 degree

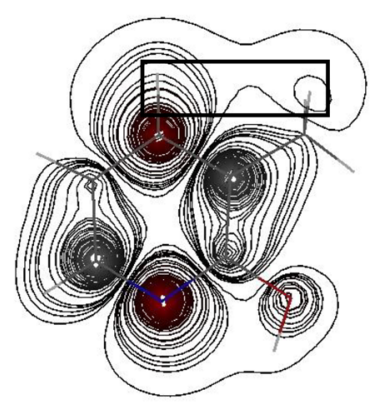

LUMO

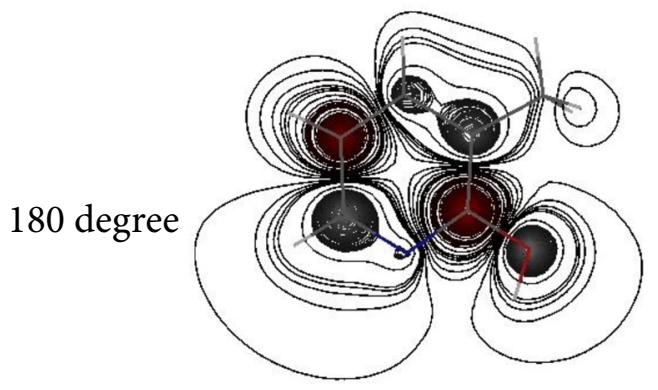

Fig. 9. Contour diagrams of the HOMO and LUMO of 3M2HP in the minimum energy conformation ( 0 degree) and top of the barrier conformation (180 degree). The contour diagrams are plotted with the same sensitivity.

in the excited state, i.e. rotation of the methyl group upon excitation from the ground electronic state to the excited electronic state $\left(S_{0} \rightarrow S_{1}\right)$.

\subsubsection{5-Methyl-2-hydroxypyridine}

As observed from the obtained REMPI spectrum, there is no such change in the conformation of the methyl group obtained in $5 \mathrm{M} 2 \mathrm{HP}$ as in $3 \mathrm{M} 2 \mathrm{HP}$ when excitation took place from the ground electronic state to the excited electronic state. The B3LYP/TZVP level of theory has been taken into account for the further investigation of the conformation and torsional potential of the molecule in the excited state $\left(\mathrm{S}_{1}\right)$. The optimized geometry of 5-methyl-2-hydroxypyridine in the $\mathrm{S}_{1}$ state is shown in Fig. 10(a). The ring frame is always planar whenever geometry optimization

(b)

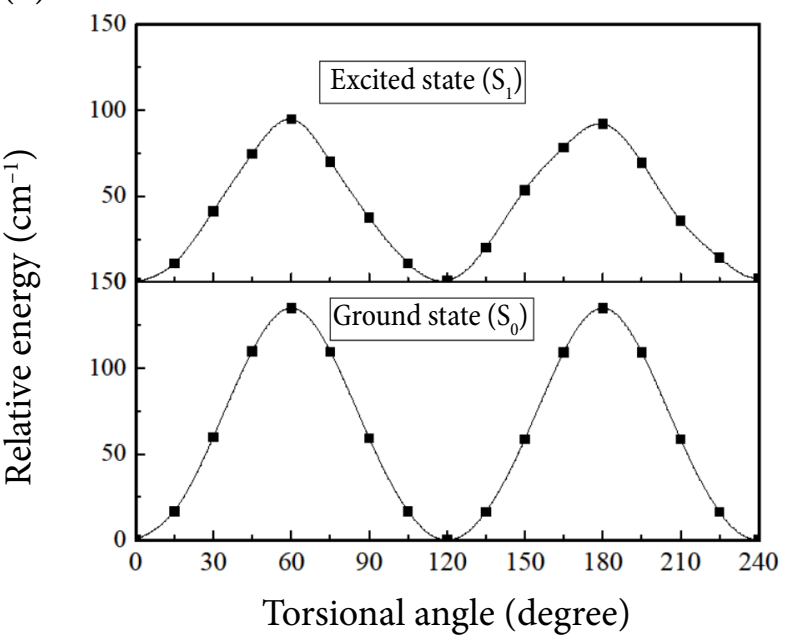

Fig. 10. (a) The optimized geometry and (b) torsional angle dependence of the potential barrier curve for 5M2HP using the B3LYP/TZVP level of theory. 


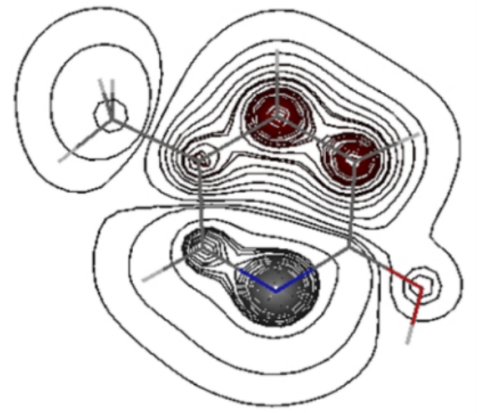

HOMO

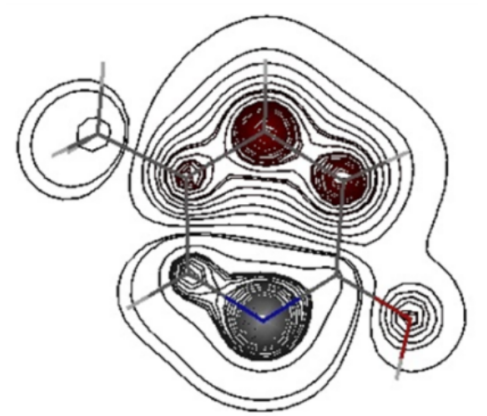

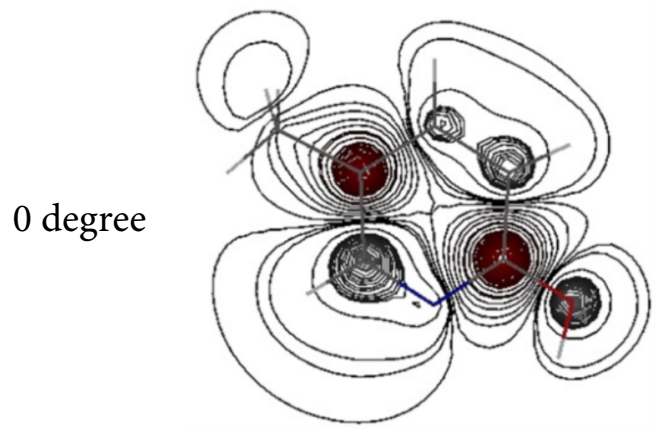

LUMO

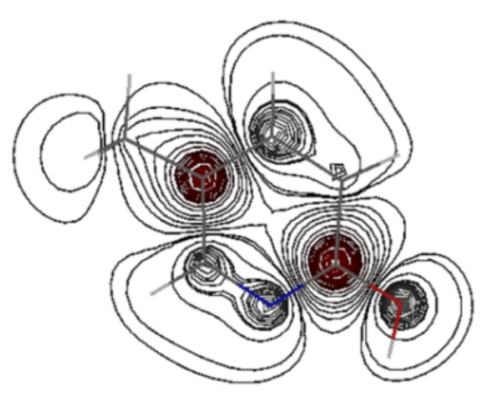

Fig. 11. Contour diagrams of the HOMO and LUMO of 5M2HP in the minimum energy conformation ( 0 degree) and top of the barrier conformation (180 degree). The contour diagrams are plotted with the same sensitivity.

in the minimum energy conformation has been performed. The torsional angle dependence of the potential energy curve for the excited state $\left(S_{1}\right)$ is shown in Fig. 10(b). The calculated three-fold potential term $V_{3}$ was found to be $92 \mathrm{~cm}^{-1}$. The obtained potential curve for the optimized geometry is in agreement with the REMPI spectrum of the molecule.

As discussed previously, the observed $\pi^{\star}-\sigma^{\star}$ hyperconjugation explained the changes in the methyl group conformation and in the magnitude of the torsional potential barrier in 3M2HP upon excitation $\left(\mathrm{S}_{0} \rightarrow \mathrm{S}_{1}\right)$. But in the case of $5 \mathrm{M} 2 \mathrm{HP}$, there is no change of methyl group conformation (as in the REMPI spectrum) hence no $\pi^{\star}-\sigma^{\star}$ hyperconjugation is involved. The orbital contour diagram for 5-methyl-2-hydroxypyridine in the HOMO and LUMO in the minimum energy conformation and top of the barrier energy conformation is shown in Fig. 11.

\section{Conclusions}

The obtained REMPI spectrum shows an internal rotation of the methyl group in 3-methyl-2-hydroxypyridine upon excitation from the ground state to the excited state which can also be observed from the obtained results from the ab initio calculations as well as from the intensity pattern calculation. The band at 33830 and $34105 \mathrm{~cm}^{-1}$ was assigned as the $\pi \pi^{\star}$ transition state and named as the origin band for $3 \mathrm{M} 2 \mathrm{HP}$ and $5 \mathrm{M} 2 \mathrm{HP}$, respectively. Many low frequency vibrational bands were near the origin band which could be because of the vibronic coupling of the $n \pi^{*}$ and $\pi \pi^{*}$ transition states in the 3-methyl-2-hydroxypyridine molecule. The $\pi^{\star}-\sigma^{\star}$ hyperconjugation is responsible for the conformational change in the methyl group in 3-methyl-2-hydroxypyridine, while there was no hyperconjugation in 5-methyl-2-hydroxypyridine.

\section{Acknowledgements}

Authors would like to thank the Department of Science and Technology, India for providing the financial support for this work. We would also like to thank Prof. T. Kundu from the Indian Institute of Technology Bombay and Dr. Rajeev K. Sinha from Manipal University, India for their valuable suggestions and continuous discussion. 


\section{Appendix 1}

Table ST1. Band position in the IR and Raman spectra of 3M2HP along with the theoretically calculated frequencies using the B3LYP/TZVP level of theory.

\begin{tabular}{|c|c|c|c|c|c|}
\hline Calculated frequencies, $\mathrm{cm}^{-1}$ & Relative IR intensity & Raman activity & $\mathrm{IR}, \mathrm{cm}^{-1}$ & Raman, $\mathrm{cm}^{-1}$ & Assignments \\
\hline \multicolumn{6}{|c|}{ In-plane vibrations } \\
\hline 286 & 3.46 & 0.63 & 285 & & $v_{11}$ \\
\hline 462 & 7.49 & 0.61 & 490 & 482 & $v_{10}$ \\
\hline 543 & 7.47 & 4.64 & 550 & & $v_{9}$ \\
\hline 607 & 1.36 & 7.45 & 586 & 588 & $v_{8}$ \\
\hline 759 & 2.32 & 20.95 & & & $v_{7}$ \\
\hline 1082 & 12.42 & 16.48 & & & $v_{6}$ \\
\hline 1359 & 78.39 & 7.19 & 1366 & 1350 & $v_{5}$ \\
\hline 3166 & 7.98 & 116.64 & 3138 & & $v_{4}$ \\
\hline 3171 & 17.16 & 66.91 & & & $v_{3}$ \\
\hline 3203 & 12.47 & 182.62 & 3267 & & $v_{2}$ \\
\hline 3755 & 81.10 & 121.32 & 3744 & & $v_{1}$ \\
\hline \multicolumn{6}{|c|}{ Out-of-plane vibrations } \\
\hline 126 & 0.13 & 0.46 & 135 & 107 & $v_{39}$ \\
\hline 163 & 0.13 & 1.20 & 162 & 150 & $v_{38}$ \\
\hline 273 & 8.88 & 1.79 & 272 & 271 & $v_{37}$ \\
\hline 441 & 8.64 & 0.01 & 449 & 421 & $v_{36}$ \\
\hline 526 & 77.60 & 2.53 & 521 & 536 & $v_{35}$ \\
\hline 564 & 14.76 & 0.81 & 557 & & $v_{34}$ \\
\hline 741 & 10.39 & 0.36 & & 748 & $v_{33}$ \\
\hline 796 & 44.91 & 0.03 & 773 & & $v_{32}$ \\
\hline 886 & 11.34 & 1.27 & 879 & 886 & $v_{31}$ \\
\hline 943 & 0.01 & 0.25 & 937 & & $v_{30}$ \\
\hline 957 & 1.59 & 0.09 & & & $v_{29}$ \\
\hline 1013 & 13.61 & 4.51 & & 1004 & $v_{28}$ \\
\hline 1063 & 1.38 & 0.09 & 1049 & 1060 & $v_{27}$ \\
\hline 1122 & 88.64 & 1.11 & 1103 & 1108 & $v_{26}$ \\
\hline 1191 & 53.34 & 4.10 & & 1165 & $v_{25}$ \\
\hline 1235 & 53.26 & 4.96 & 1221 & 1261 & $v_{24}$ \\
\hline 1306 & 13.05 & 1.32 & & & $v_{23}$ \\
\hline 1319 & 26.98 & 16.56 & & 1318 & $v_{22}$ \\
\hline 1426 & 3.22 & 10.12 & 1423 & & $v_{21}$ \\
\hline 1466 & 87.23 & 5.40 & 1472 & 1460 & $v_{20}$ \\
\hline 1481 & 7.67 & 10.51 & & 1483 & $v_{19}$ \\
\hline 1501 & 58.66 & 3.16 & & 1539 & $v_{18}$ \\
\hline 1509 & 39.28 & 2.00 & & 1569 & $v_{17}$ \\
\hline 1631 & 73.49 & 11.85 & & 1622 & $v_{16}$ \\
\hline 1641 & 18.99 & 23.85 & & & $v_{15}$ \\
\hline 3034 & 22.91 & 211.57 & & & $v_{14}$ \\
\hline 3084 & 12.23 & 80.28 & 3074 & & $v_{13}$ \\
\hline 3111 & 16.25 & 61.48 & & & $v_{12}$ \\
\hline
\end{tabular}




\section{Appendix 2}

Table ST2. Band position in the IR and Raman spectra of 5M2HP along with the theoretically calculated frequencies using the B3LYP/TZVP level of theory.

\begin{tabular}{|c|c|c|c|c|c|}
\hline Calculated frequencies, $\mathrm{cm}^{-1}$ & Relative IR intensity & Raman activity & IR, $\mathrm{cm}^{-1}$ & Raman, $\mathrm{cm}^{-1}$ & Assignments \\
\hline \multicolumn{6}{|c|}{ In-plane vibrations } \\
\hline 306 & 0.95 & 0.28 & 311 & 303 & $v_{8}$ \\
\hline 438 & 19.34 & 0.29 & 438 & & $v_{7}$ \\
\hline 492 & 0.22 & 8.84 & 500 & & $v_{6}$ \\
\hline 666 & 4.77 & 5.18 & & 661 & $v_{5}$ \\
\hline 759 & 3.84 & 1.65 & 758 & 761 & $v_{4}$ \\
\hline 877 & 8.88 & 32.44 & & 858 & $v_{3}$ \\
\hline 3210 & 3.12 & 135.99 & & & $v_{2}$ \\
\hline 3756 & 80.28 & 131.05 & 3740 & & $v_{1}$ \\
\hline \multicolumn{6}{|c|}{ Out-of-plane vibrations } \\
\hline 74 & 0.09 & 0.60 & 77 & & $v_{39}$ \\
\hline 141 & 0.60 & 0.12 & 145 & & $v_{38}$ \\
\hline 322 & 0.37 & 1.83 & 320 & & $v_{37}$ \\
\hline 430 & 0.02 & 0.28 & 428 & & $v_{36}$ \\
\hline 486 & 100.16 & 1.71 & 478 & 488 & $v_{35}$ \\
\hline 546 & 23.35 & 1.28 & & & $v_{34}$ \\
\hline 731 & 4.59 & 1.11 & & & $v_{33}$ \\
\hline 828 & 32.86 & 0.22 & & & $v_{32}$ \\
\hline 923 & 3.24 & 0.25 & & & $v_{31}$ \\
\hline 972 & 0.02 & 0.11 & 972 & & $v_{30}$ \\
\hline 1006 & 0.06 & 1.84 & & 1016 & $v_{29}$ \\
\hline 1045 & 9.14 & 0.58 & 1053 & & $v_{28}$ \\
\hline 1063 & 2.38 & 0.04 & & & $v_{27}$ \\
\hline 1151 & 19.12 & 1.86 & 1144 & 1154 & $v_{26}$ \\
\hline 1188 & 200.04 & 3.06 & & & $v_{25}$ \\
\hline 1241 & 1.74 & 9.88 & 1232 & 1249 & $v_{24}$ \\
\hline 1309 & 15.26 & 8.65 & & 1298 & $v_{23}$ \\
\hline 1319 & 84.00 & 9.75 & & & $v_{22}$ \\
\hline 1368 & 38.60 & 3.82 & 1364 & 1359 & $v_{21}$ \\
\hline 1425 & 1.12 & 17.24 & 1427 & 1415 & $v_{20}$ \\
\hline 1438 & 14.88 & 3.05 & & 1453 & $v_{19}$ \\
\hline 1490 & 7.20 & 11.85 & & 1488 & $v_{18}$ \\
\hline 1505 & 11.85 & 11.49 & & & $v_{17}$ \\
\hline 1526 & 209.95 & 0.16 & 1549 & 1546 & $v_{16}$ \\
\hline 1626 & 35.94 & 7.98 & 1614 & 1628 & $v_{15}$ \\
\hline 1652 & 76.15 & 27.84 & 1661 & 1656 & $v_{14}$ \\
\hline 3028 & 34.27 & 239.38 & 3032 & & $v_{13}$ \\
\hline 3075 & 17.36 & 91.46 & & & $v_{12}$ \\
\hline 3104 & 14.97 & 59.82 & & & $v_{11}$ \\
\hline 3152 & 24.05 & 102.11 & 3128 & & $v_{10}$ \\
\hline 3168 & 10.02 & 83.36 & & & $v_{9}$ \\
\hline
\end{tabular}




\section{References}

[1] G.A. Bickel, G.W. Leach, D.R. Demmer, J.W. Hager, and S.C. Wallace, The torsional spectra of jet-cooled methyl substituted indoles in the ground and first excited states, J. Chem. Phys. 88, 1-8 (1988), https://doi.org/10.1063/1.454636

[2] W.A. Wassam and E.C. Lim, 'Proximity effect' and radiationless transitions in aromatic molecules with non-bonding electrons, J. Mol. Struc. 47, 129-198 (1978), https://doi.org/10.1016/00222860(78)87184-1

[3] A. Hiraya, Y. Achiba, K. Kimura, and E.C. Lim, Identification of the lowest energy $n \pi^{\star}$ states in gas-phase polycyclic monoazines: Quinoline and isoquinoline, J. Chem. Phys. 81(7), 3345-3347 (1984), https://doi.org/10.1063/1.447998

[4] G. Fischer and A.E.W. Knight, Narrow band laser excited fluorescence as a probe of the near-resonance vibronic coupling in isoquinoline vapour, Chem. Phys. 17(3), 327-342 (1976), https://doi. org/10.1016/S0301-0104(76)80035-3

[5] G. Fischer and R. Naaman, Near resonance vibronic coupling. Isoquinoline, Chem. Phys. 12(4), 367-379 (1976), https://doi.org/10.1016/03010104(76)87075-9

[6] P.M. Felker and A.H. Zewail, Jet spectroscopy of isoquinoline, Chem. Phys. Lett. 94(5), 448-453 (1983), https://doi.org/10.1016/00092614(83)85030-1

[7] A.K. Jameson, B.E. Forch, K.T. Chen, S. Okajima, H. Saigusa, and E.C. Lim, Electronic and vibrational relaxations in van der Waals complexes of isoquinoline with argon and nitrogen: energetics and dynamics of photodissociation leading to $\mathrm{S}_{1}\left(n \pi^{\star}\right)$ isoquinoline, J. Phys. Chem. 88(21), 4937-4943 (1984), https://doi.org/10.1021 $1150665 \mathrm{a} 029$

[8] R.K. Sinha, B.P. Singh, and T. Kundu, Effect of electronic states coupling on methyl torsion in 3-methylisoquinoline, Indian J. Phys. 86(3), 187194 (2012), https://doi.org/10.1007/s12648-0120040-3
[9] A.K. Srivastava, R.K. Sinha, S. Saxena, and T. Kundu, Effect of methylation on 2-hydroxypyridine in ground state: Theoretical study, Int. J. Chem. Sci. 16(2), 270(1-13) (2018), https://doi. org/10.21767/0972-768X.1000270

[10]M.J. Frisch, G.W. Trucks, H.B. Schlegel, G.E. Scuseria, M.A. Robb, J.R. Cheeseman, G. Scalmani, V. Barone, B. Mennucci, G.A. Petersson, et al., Gaussian09 Revision E.01 (Gaussian Inc., Wallingford, CT, 2009).

[11]A.R. Allouche, Gabedit - A graphical user interface for computational chemistry softwares, J. Comp. Chem. 32(1), 174-182 (2011), https:// doi.org/10.1002/jcc.21600

[12] R.K. Sinha, B.P. Singh, and T. Kundu, Origin of threefold methyl torsional potential in methylindoles, Theo. Chem. Acc. 121(1), 59-70 (2008), https://doi.org/10.1007/s00214-008-0450-7

[13]Y. Matsuda, T. Ebata, and N. Mikami, IR-UV double-resonance spectroscopic study of 2-hydroxypyridine and its hydrogen-bonded clusters in supersonic jets, J. Phys. Chem. A. 105(14), 3475-3480 (2001), https://doi.org/10.1021 jp003272x

[14]M.R. Nimlos, D.F. Kelley, and E.R. Bernstein, Spectroscopy, structure, and proton dynamics of 2-hydroxypyridine and its clusters with water and ammonia, J. Phys. Chem. 93(2), 643-651 (1989), https://doi.org/10.1021/j100339a030

[15]B. Pradhan, R.K. Sinha, B.P. Singh, and T. Kundu, Origin of methyl torsional barrier in 1-methyl-2(1H)-pyridinimine and 3-methyl2(1H)-pyridone: II. Ground state, J. Chem. Phys. 126(11), 114313(1-8) (2007), https://doi. org/10.1063/1.2566602

[16]H. Nakai and M. Kawai, $\pi^{\star}-\sigma^{\star}$ hyperconjugation mechanism on the rotational barrier of the methyl group (I): Substituted toluenes in the ground, excited, and anionic states, J. Chem. Phys. 113(6), 2168-2174 (2000), https://doi. org/10.1063/1.482029 
METILO GRUPĖS META PAKEITIMO 2-HIDROKSIPIRIDINE POVEIKIS: SPEKTROSKOPINIS TYRIMAS

\author{
A.K. Srivastava ${ }^{a}$, S. Saxena ${ }^{b}$ \\ a Indijos Bombëjaus technologiju institutas, Mumbajus, Maharaštra, Indija

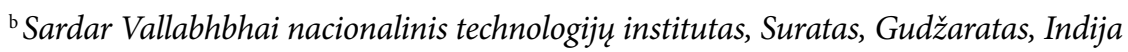

